\title{
„Ein wichtiger gesellschaftlicher Beitrag“
}

Bracco Imaging ist seit November 2014 Premiumpartner der Geburtshaus Wilhelm Conrad Röntgen Stiftung. Geschäftsführer Dr. Thilo Schneider skizziert die Motive seines Unternehmens für diese Partnerschaft

? Herr Dr. Schneider, Sie konnten sich am 25.11. bei Ihrem Besuch in Lennep selbst ein Bild von der Baustelle Geburtshaus machen - Ihr Eindruck?

Ich war zunächst gefangen von der Geschichte des Hauses, die mit dem Entdecker der Strahlung, die Wissenschaft und Medizin so grundlegend verändert hat, verbunden ist. Man muss an diesem Ort zunächst an die Lebensleistung von Röntgen denken, auch wenn er nur für wenige Jahre in Lennep gelebt hat. Weiter beeindruckte mich das sichtbare Engagement der Stiftung, dieses historische Gebäude sachgerecht zu sanieren und der Öffentlichkeit zugänglich zu machen. Schon heute ist zu erkennen, dass diese Restaurierung mit großem Sachverstand und der
Liebe zum Detail durchgeführt wird. Ich wünsche diesem Vorhaben ein gutes Gelingen.

? Bracco engagiert sich als Premiumpartner für den Wiederaufbau des HauseS. Aus welcher Motivation heraus engagiert sich Ihr Unternehmen für das Geburtshaus Wilhelm Conrad Röntgens?

Bracco ist eines der weltweit führenden Unternehmen im Bereich der bildgebenden Diagnostik. Die Arbeiten von Wilhelm Conrad Röntgen bilden Basis und Ausgangspunkt für eine rasante und revolutionäre Entwicklung bildgebender Diagnostikverfahren. Bracco begleitet diese Entwicklung mit innovativen Kontrastmitteln seit vielen Jahrzehnten und ver-

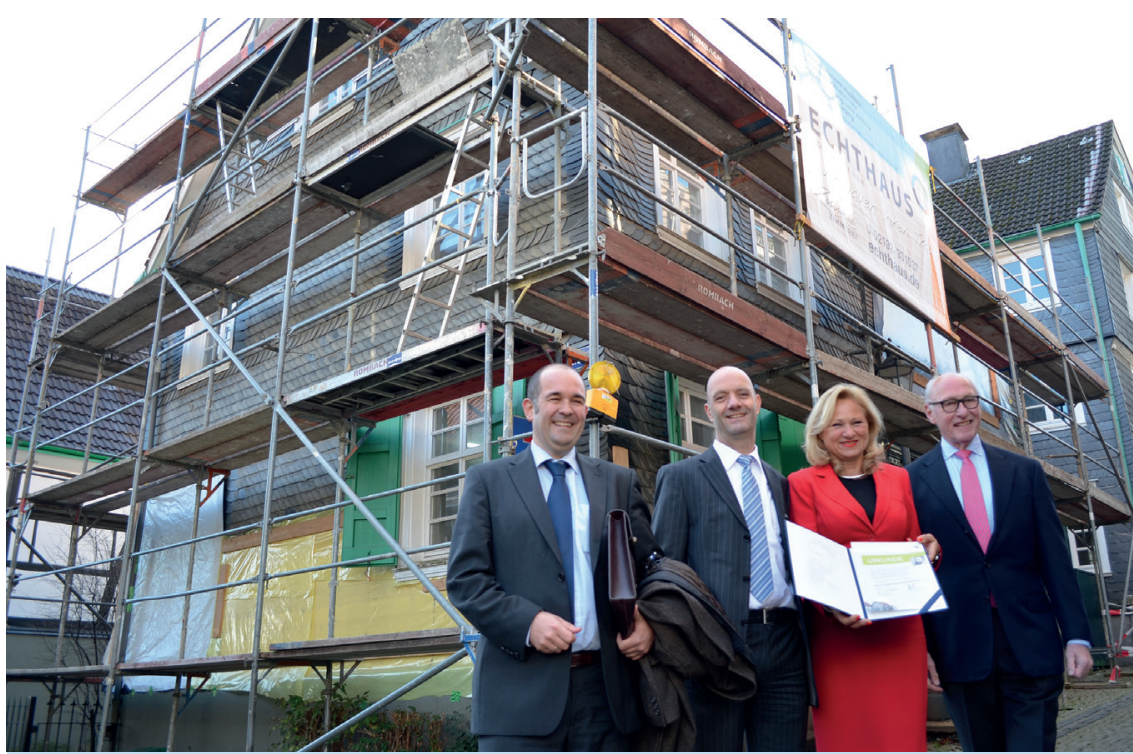

Bracco Imaging ist neuer Premiumpartner der Geburtshaus-Stiftung. Herr Stegmüller, Herr Dr. Schneider und Frau Middelhof von der Firma Bracco sowie Prof. Mödder (v.l.n.r.) bei der Urkundenverleihung. (Foto: Falkenberg) steht sich als Teil der wissenschaftlichen Community, die als Erben von Wilhelm Conrad Röntgen diese Verfahren weiterentwickeln. Es ist uns auch immer ein Anliegen gewesen, historische Bezüge aufrecht zu erhalten und außergewöhnliche, innovative Menschen zu ehren. Unser Beitrag zum Wiederaufbau des Geburtshauses von Wilhelm Conrad Röntgen steht in dieser Tradition des Unternehmens.

? Welche Ideen des Nutzungskonzepts sprechen Sie besonders an? Ich kann mich eigentlich mit allen Aspekten des Nutzungskonzeptes identifizieren.Der Erhalt des Gebäudes ist kulturund wissenschaftshistorisch wünschenswert und wird von uns begrüßt. Das Gebäude der Öffentlichkeit zugänglich zu machen und dabei an einen großen Mann der Naturwissenschaften zu erinnern, ist ein wichtiger gesellschaftlicher Beitrag, den die Stiftung leistet. Der zukunftsorientierte Aspekt des wissenschaftlichen Begegnungszentrums im Geburtshaus von Röntgen kann aus unserer Sicht ein wichtiger Beitrag werden, die bildgebende Diagnostik mit neuen Impulsen zu versorgen. Wir wünschen uns, dass dieses Vorhaben gelingt und unterstützen es deshalb auch substanziell.

? Sie möchten Ihrerseits den Außendienst Ihres Unternehmens zu „Botschaftern“ der Spendenkampagne machen und das Geburtshaus in Ihre Unternehmenskommunikation einbinden - verraten Sie Details? Bracco trifft als Unternehmen mit vielen Ärzten, Fachpersonal und Institutionen zusammen, die im Bereich der bildgebenden Diagnostik tätig sind. Es bietet sich einfach an, dass die Bracco-Mitarbeiter über das Engagement ihres Unternehmens bei der Wiederherstellung des Geburtshauses von Wilhelm Conrad Röntgen sprechen und damit möglichst viele weitere Sponsoren für dieses Vorhaben gewinnen. 